\title{
Association of Intima-Media Thickness of Carotid Arteries With Remnant Lipoproteins in Men and Women
}

\author{
J. PIŤHA ${ }^{1}$, J. KOVÁŘ ${ }^{1}$, Z. ŠKODOVÁ ${ }^{1}$, R. CÍFKOVÁ ${ }^{2}$, P. STÁVEK ${ }^{1}$, L. ČERVENKA ${ }^{1}$, \\ T. ŠEJDA ${ }^{3}$, V. LÁNSKÁ ${ }^{4}$, R. POLEDNE ${ }^{1}$ \\ ${ }^{1}$ Center for Experimental Medicine, Institute for Clinical and Experimental Medicine, Prague, \\ Czech Republic, ${ }^{2}$ Center for Cardiovascular Prevention, First Faculty of Medicine, Charles \\ University in Prague and Thomayer Hospital, Prague, Czech Republic, ${ }^{3}$ Department of Angiology, \\ Prague, Czech Republic, ${ }^{4}$ Deparment of Statistics, Institute for Clinical and Experimental Medicine, \\ Prague, Czech Republic
}

Received September 21, 2015

Accepted October 5, 2015

\section{Summary}

The subclass of triglyceride-rich lipoproteins - remnant-like particles (RLP) seems to be strong and independent risk factor for cardiovascular disease. We evaluated the role of RLP and other risk factors (RF) with sonographically measured intimamedia thickness of carotid arteries (IMT CCA) in a cohort of Czech population including women defined according to the time after menopause. We investigated relation of IMT CCA to age, weight, central obesity, plasma lipids including remnant-like particles cholesterol (RLP-C) and triglycerides (RLP-TG) in 136 men and 160 women. Using multiple linear regression analysis, significant association between IMT CCA and RLP-C was found in women 1-7 years after menopause. In the whole group of women, only age and fasting blood glucose were independently associated with IMT CCA. In men only age significantly correlated with IMT CCA. Significant decrease of all plasma lipids between 1988 and 1996 in men was detected, while in women significant increase in triglycerides and no change in non-HDL cholesterol was observed. RLP-C was the strongest independent RF for atherosclerosis in postmenopausal women but its association with IMT CCA was limited to several years after menopause. In conclusion, women changing reproductive status could be more sensitive to atherogenic impact of remnant lipoproteins.

\section{Key words}

Remnant lipoproteins - Preclinical atheroslerosis - Sex • Reproductive status

\section{Corresponding author}

J. Pit'ha, Laboratory for Atherosclerosis Research, Center for Experimental Medicine, Institute for Clinical and Experimental Medicine, Vídeňská 1958/9, 14021 Prague, Czech Republic. E-mail: japi@ikem.cz

\section{Introduction}

The LDL particles, carrying most of the total cholesterol circulating in the plasma are considered the most atherogenic of the lipoproteins and are nowadays the prime target in lowering plasma cholesterol. However, elevated LDL cholesterol does not explain all cardiovascular events, and among other risk factors (RF) triglycerides are repeatedly and extensively investigated as an independent RF for cardiovascular diseases (CVD) (Hokanson and Austin 1996, Austin 1999, Libby 2015, Nordestgaard and Varbo 2014). In observational epidemiological studies the association of triglycerides has been proved as an independent risk factor for CVD in patients with (Jeppesen et al. 1998) and without (Miller et al. 1998) coronary heart disease, but data proving clear benefit from triglycerides reduction are missing.

One explanation for these controversies is the high variability of triglyceride concentration. Another explanation is close connection of triglycerides with other cardiovascular risk factors (CV RF) (low HDL cholesterol, small LDL particles, insulin resistance, thrombogenic factors). 
One of the most widely accepted hypothesis suggests triglycerides reflect increased concentration and/or inadequate postprandial catabolism of atherogenic triglyceride-rich lipopoproteins. The possible atherogenity of triglyceride-rich lipoproteins connected with postprandial lipemia has been intensively discussed for a long time (Havel 1994, Varbo et al. 2014).

Methodology has been developed, measuring partially hydrolyzed lipoprotein remnants of both intestinal and hepatic triglyceride-rich lipoproteins (Nakajima et al. 1996). These triglyceride-rich lipoproteins called remnant-like particles (RLP) have been already proved to be an independent risk factor for recurrent coronary events (Kugiyama et al. 1999) and sudden cardiac death (Takeichi et al. 1997). Another evidence for atherogenic properties of RLP, came from studies describing deleterious effect of RLP on endothelial function (Inue et al. 1998), or IMT CCA measured by utrasound (Boquist et al. 1999, Karpe et al. 2001). Recent studies used so called nonHDL/nonLDL cholesterol obtained mainly from nonfasting blood and calculated as total cholesterol minus LDL cholesterol minus HDL cholesterol; this parameter reliably reflects cholesterol content in remnant lipoprotein particles and is also strongly associated with cardiovascular events (Varbo et al. 2014)

In observational studies, the association between triglycerides and CVD is strongest in women (Castelli 1992, Bengston et al. 1993). In women from the Framingham study, cholesterol in RLP (RLP-C) was the strongest independent predictor for coronary events (McNamara et al. 2001). However, no data are available about different impact of RLP on atherosclerosis in women, stratified according to the time after menopause. However, at the first years after menopause sudden hormonal changes can modify the properties of the artery wall, which can become less resistant to certain RF (Pitha et al. 2013).

Therefore we addressed two questions in our retrospective observational study. First, if there are any relations between RLP and other CV RF with atherosclerosis in the Czech population with high prevalence of hypertriglyceridemia (Cífková et al. 2010). Second, if these relations are different in postmenopausal women at different time after menopause. For measurements of remnant lipoproteins we used the technique introduced by Nakajima (Nakajima et al. 1996).

\section{Methods}

\section{Population}

In 1988, $1 \%$ representative sample of the Czech population from one district (Benešov) was examined for CV RF according to the protocol of the MONICA study. In 1996, all participants were invited for re-examination by similar protocol. Data were obtained in 296 persons (136 men and 160 women aged 33-72 years). Respondence rate was $74.2 \%$.

To analyze the impact of menopause and its duration on relations between IMT CCA and CV RF, women were divided into three groups. Women premenopausal in 1988 and 1996 were defined as premenopausal (PremW). Women premenopausal in 1988 but at least for one year postmenopausal in 1996 were defined as newly postmenopausal (NewPostmW). Finally, women postmenopausal both in 1988 and 1996 were defined as postmenopausal (PostmW). The local ethical committee approved the study and all participants gave their informed consent.

\section{Risk factors}

Following RF were measured: body mass index (BMI), calculated as weight in $\mathrm{kg} /(\text { height in } \mathrm{m})^{2}$, central obesity calculated as waist to hip ratio (WHR) and waist circumference (W), systolic (SBP) and diastolic (DBP) blood pressures, calculated as a mean of three measurements at one visit using a mercury sphygmomanometer.

Information on the presence of CVD was obtained by interview. The history of ischemic heart disease and stroke was recorded in each participant. Current smoking was defined as smoking regularly at least 3 months before examination. Hypertension was defined as SBP >140 mm Hg and/or DBP >90 mm Hg, use of antihypertensive drugs, history of hypertension, or all of these. Diabetes mellitus was defined as fasting bloood glucose (FBG) $>7.0 \mathrm{mmol} / \mathrm{l}$, use of antidiabetic drugs, history of diabetes, or all of these. In women, information on the age of menopause, hysterectomy and hormone replacement therapy was recorded.

Blood was drawn after a $12 \mathrm{~h}$ overnight fast into tubes containing EDTA. Plasma was separated by centrifugation. Plasma lipid and lipoprotein concentrations - total cholesterol (TChol), triglycerides (TG), and cholesterol in high-density lipoproteins (HDLC) were analyzed using Boehringer and Hoffman LaRoche reagent kits. Non-HDL cholesterol (NHDLC) 
was calculated as TChol - HDLC. Cholesterol (RLP-C) and triglyceride (RLP-TG) concentrations in apo-E rich remnant lipoprotein particles were measured by combination of 2 monoclonal antibodies against ApoB 100 and apoA1 as already published (Nakajima et al. 1996). FBG was measured by Lachema kit.

To compare changes of RF between 1988 and 1996, following RF were analyzed: BMI, WHR, SBP, DBP, TChol, TG, HDLC, NHDLC. In 1988 FBG and RLP were not measured.

As a surrogate endpoint for atherosclerosis we used intima-media thickness in common carotid arteries (IMT CCA).

\section{Ultrasound measurements}

Measurements of IMT CCA were performed on Acuson 128/4 ultrasound machine (7.0 MHz probe). Longitudinal images of the left and right common carotid artery were recorded on videotapes. The segment $1 \mathrm{~cm}$ proximal to the bulb was selected for measurements of the far wall. Measurements were performed in $1 \mathrm{~mm}$ distances, off-line, in digitalized picture on the computer, using ImageMeasure PC Vision Microscience software (Imaging Technology Inc., Bedford, MA, USA). In each segment of common carotid artery 10 measurements were obtained. For statistical analyses was used the mean of all 20 measurements from right and left carotid arteries (right + left carotid arteries/2). This approach was minor modification of the methods described previously (Pitha et al. 1999, 2013). Carotid ultrasound measurements and computer readings were done by one physician (JP) blinded to all data. Variability was assessed by repeated examination in 20 randomly chosen participants (10 men and 10 women, average age 52 years). Intraclass correlation coefficient was 0.97 , (95\% CI $0.93,0.99)$. Maximal and minimal differences were 0.3 and $0.001 \mathrm{~mm}$ respectively.

\section{Statistical analyses}

Data were analyzed as categorical values or means with standard deviations. In evaluation of RF with skewed distribution, log-transformation was used. In the case of means, differences between groups were tested by the two-tailed t-test. The chi-squared test was used for comparison of proportions. The differences in selected RF between 1988 and 1996 were calculated by paired t-test. Univariate correlation between IMT CCA and risk factors was done by Spearman correlation test. Because significant correlation between IMT CCA and age was found, all correlations were standardized for age. Linear multiple regression analysis was used to evaluate independent association between IMT CCA and RF.

\section{Results}

\section{Baseline data}

Baseline data are shown in Table 1.

In 1996, men had significantly higher WHR, DBP and RLP-TG than women. Women had significantly higher HDLC than men. No differences between men and women were found in age, IMT CCA, BMI, SBP, TChol, TG, RLP-C and FBG.

Men smoked more frequently (36\%) than women $(17 \%)$. This difference was statistically significant $(p<0.01)$. No significant differences between men and women were found in prevalence of CVD (12 and $8 \%$ ), diabetes mellitus (15 and $11 \%)$, hypertension (62 and $61 \%$ ), use of antihypertensive drugs (27 and $33 \%$ ), use of hypolipemic drugs (5 and $3 \%$ ), and use of acetylsalicylic acid (10 and $6 \%$ ).

Across all three groups of women defined according to their menopausal status (PremW, NewPostmW and PostmW) we detected significant trend for decrease of HDLC and increase in all RF with the exception of non-significant trend in DBP.

In PremW, NewPostmW and PostmW was the prevalence of diabetes mellitus $0 \%, 9 \%$ and $21 \%$ (p for trend $<0.01$ ), hypertension $38 \%, 61 \%$ and $81 \%$ (p for trend $<0.01$ ), use of antihypertensive drugs $8 \%$, $42 \%$ and $48 \%$ ( $\mathrm{p}$ for trend $<0.01$ ). The prevalence of CVD ( $4 \%, 9 \%$ and $11 \%)$, use of hypolipemics ( $0 \%$, $9 \%$ and $2 \%)$ and use of acetylsalicylic acid $(2 \%, 6 \%$ and $10 \%$ ) were not different among these three groups.

\section{Changes in risk factors between 1988 and 1996}

Changes in risk factors between 1988 and 1996 are shown in Table 2.

Both in men and women, significantly increased BMI and significantly decreased TChol and HDLC. NHDLC significantly decreased in men, but not in women. TG did not change significantly in men, but significantly increased in women. WHR significantly increased in men, while there was no significant change in women.

Between 1988 and 1996 smoking significantly decreased in men $(9 \%, \mathrm{p}<0.01)$, but not in women $(1 \%$, $\mathrm{p}=n$.s.). Difference in change of smoking habits between men and women was statistically significant $(p<0.05)$. 
Table 1. Baseline characteristics of men and women from population sample.

\begin{tabular}{|c|c|c|c|c|c|}
\hline & $\begin{array}{c}\text { Men } \\
n=136\end{array}$ & $\begin{array}{c}\text { Women } \\
n=160\end{array}$ & $\begin{array}{c}\text { PremW } \\
n=53\end{array}$ & $\begin{array}{c}\text { NewPostmW } \\
n=32\end{array}$ & $\begin{array}{c}\text { PostmW } \\
n=62\end{array}$ \\
\hline Age, years & $53.3(11)$ & & $42.2(6)$ & $53.8(6)$ & $64.1(6)$ \\
\hline$I M T C C A, m m$ & $0.623(0.2)$ & $0.614(0.1)$ & $0.529(0.1)$ & $0.598(0.1)$ & $0.697(0.2)$ \\
\hline$B M I, \mathrm{~kg} / \mathrm{m}^{2}$ & $28.3(4.0)$ & $29.2(5.7)$ & $27.6(5.2)$ & $30.8(5.7)$ & $30.2(5.8)$ \\
\hline WHR & $0.97(0.08)$ & $0.84(0.07)^{* * *}$ & $0.80(0.07)$ & $0.86(0.05)$ & $0.86(0.07)$ \\
\hline$W, \mathrm{~cm}$ & $99.6(10)$ & $90.8(13)^{* * *}$ & $84.6(12.4)$ & $94.8(13.6)$ & $94.3(12.4)$ \\
\hline$S B P, m m ~ H g$ & $140.8(19.2)$ & $139.9(22.9)$ & $128.3(16.3)$ & $142.9(24.1)$ & $149.3(22.9)$ \\
\hline $\mathrm{DBP}, \mathrm{mm} \mathrm{Hg}$ & $87.1(10.4)$ & $84.6(11.2)^{*}$ & $82.6(9.5)$ & $85.6(14.0)$ & $86.0(11.2)$ \\
\hline TChol, mmol/l & $5.43(1.1)$ & $5.59(1.2)$ & $5.15(1.1)$ & $5.56(0.9)$ & $6.02(1.2)$ \\
\hline$T G, \mathrm{mmol} / \mathrm{l}$ & $2.18(1.8)$ & $1.86(1.2)$ & $1.39(0.8)$ & $1.76(0.9)$ & $2.35(1.5)$ \\
\hline HDLC, mmol/l & $1.21(0.3)$ & $1.44(0.4)^{* * *}$ & $1.56(0.4)$ & $1.42(0.3)$ & $1.34(0.3)$ \\
\hline NHDLC, mmol/l & $4.2(1.2)$ & $4.2(1.2)$ & $3.6(1.1)$ & $4.1(0.9)$ & $4.7(1.2)$ \\
\hline$R L P-C, \mathrm{mmol} / \mathrm{l}$ & $0.3(0.5)$ & $0.2(0.3)$ & $0.2(0.1)$ & $0.2(0.1)$ & $0.4(0.4)$ \\
\hline$R L P-T G, \mathrm{mmol} / \mathrm{l}$ & $0.4(1.0)$ & $0.2(0.4)^{* * *}$ & $0.1(0.1)$ & $0.2(0.1)$ & $0.3(0.6)$ \\
\hline$F B G, \mathrm{mmol} / \mathrm{l}$ & $6.4(2.4)$ & $6.0(1.7)$ & $5.37(0.6)$ & $5.79(0.7)$ & $6.83(2.4)$ \\
\hline
\end{tabular}

Data are expressed as means (SD). * men vs. all women $\mathrm{p}<0.05, * *$ men vs. all women $\mathrm{p}<0.01, * * *$ men vs. all women $\mathrm{p}<0.001$. IMT CCA - intima-media thickness of common carotid arteries, BMI - body mass index, WHR - waist to hip ratio, W - waist, SBP - systolic blood pressure, DBP - diastolic blood pressure, TChol - total cholesterol, TG - triglycerides, HDLC - HDL cholesterol, NHDLC - non-HDL cholesterol, RLP-C - remnant like particles cholesterol, RLP-TG - remnant like particles triglycerides, FBG - fasting blood glycemia.

Table 2. Changes in risk factors 1988-1996.

\begin{tabular}{llllll}
\hline & \multicolumn{1}{c}{$\begin{array}{c}\text { Men } \\
\mathbf{n = 1 2 6}\end{array}$} & $\begin{array}{c}\text { Women all } \\
\mathbf{n = 1 3 4}\end{array}$ & $\begin{array}{c}\text { PremW } \\
\mathbf{n = 5 0}\end{array}$ & $\begin{array}{c}\text { NewPostmW } \\
\mathbf{n}=\mathbf{2 9}\end{array}$ & $\begin{array}{c}\text { PostmW } \\
\mathbf{n = 5 5}\end{array}$ \\
\hline BMI, $\mathrm{kg} / \mathrm{m}^{2}$ & $0.7(2)^{* * *}$ & $1.1(3)^{* * *}$ & $1.8(3)^{* * *}$ & $1.5(2)^{* *}$ & $0.5(3)^{* *}$ \\
$W H R \neq$ & $0.03(0.1)^{* *}$ & $-0.01(0.08)$ & $-0.01(0.1)$ & $0.01(0.1)$ & $-0.01(0.1)$ \\
$W, \mathrm{~cm}$ & $2.3(7.3)$ & $-0.16(10.4)$ & $-0.5(9.2)$ & $1.1(8.8)$ & $-0.8(12.5)$ \\
$T C h o l, \mathrm{mmol} / \mathrm{l} \neq$ & $-0.7(1)^{* * *}$ & $-0.3(1)^{* *}$ & $-0.3(0.7)^{* *}$ & $-0.4(0.8)^{* *}$ & $-0.3(1.3)^{* *}$ \\
$T G, \mathrm{mmol} / \mathrm{l} \neq$ & $-0.3(3)$ & $0.4(1)^{* *}$ & $0.2(0.7)^{* *}$ & $0.3(0.9)^{* * *}$ & $0.6(1.3)^{* * *}$ \\
$\mathrm{HDLC}, \mathrm{mmol} / \mathrm{l}$ & $-0.12(0.2)^{* * *}$ & $-0.09(0.3)^{* * *}$ & $-0.04(0.4)^{*}$ & $-0.2(0.3)^{* * *}$ & $-0.1(0.3)^{* * *}$ \\
NHDLC, $\mathrm{mmol} / \mathrm{l} \neq$ & $-0.6(1)^{* * *}$ & $-0.2(0.9)$ & $-0.3(0.7)^{*}$ & $-0.3(0.8)$ & $-0.2(1.2)$ \\
\hline
\end{tabular}

Data are expressed as mean differences (SD). * p $<0.05, * * p<0.01, * * * p<0.001$. $\neq$ significant difference in change of risk factors between men and women $(p<0.05)$. BMI - body mass index, WHR - waist to hip ratio, W - waist, TChol - total cholesterol, TG triglycerides, HDLC - HDL cholesterol, NHDLC - non-HDL cholesterol.

In all three defined subgroups of women between 1988 and 1996, almost all variables changed in the same direction as in the whole women group. The only exception was the absence of change in DBP and NHDLC in NewPostmW and PostmW (Table 2).

\section{Differences between respondents and non-respondents}

Looking at data from 1988, non-respondents $(n=111)$ had higher SBP $(130.8+18.0$ vs. $137.7+21.8$ $\mathrm{mm} \mathrm{Hg}, \mathrm{p}<0.01)$ and higher prevalence of current smoking (44.1 vs. $28.6 \%, \mathrm{p}<0.01)$ than respondents. No differences were found in age, sex, DBP, plasma lipids, BMI, WHR and W (data not shown).

\section{Univariate correlation of IMT CCA with risk factors}

Univariate correlations of IMT CCA with risk factors are shown in Table 3.

In men and women, IMT CCA significantly correlated with age. In men, after age-adjustment, IMT CCA significantly correlated with FBG, in women with BMI, WHR, TG and FBG. In women IMT CCA significantly inversely correlated with HDLC. 
Table 3. Association of risk factors with IMT CCA, univariate analysis.

\begin{tabular}{llcccc}
\hline & $\begin{array}{c}\text { Men } \\
\mathbf{n = 1 3 6}\end{array}$ & $\begin{array}{c}\text { Women all } \\
\mathbf{n = 1 5 8}\end{array}$ & $\begin{array}{c}\text { PremW } \\
\mathbf{n = 5 3}\end{array}$ & $\begin{array}{c}\text { NewPostmW } \\
\mathbf{n = 3 2}\end{array}$ & $\begin{array}{c}\text { PostmW } \\
\mathbf{n = 6 2}\end{array}$ \\
\hline Age $\neq$ & $0.53^{* * *}$ & $0.60^{* * *}$ & $0.43^{* *}$ & $0.69 * * *$ & 0.17 \\
BMI & 0.06 & $0.160^{*}$ & 0.05 & 0.14 & $0.28^{*}$ \\
$W H R$ & -0.20 & $0.22^{*}$ & $0.35^{*}$ & $0.43^{*}$ & 0.13 \\
$W$ & 0.1 & $0.22^{* *}$ & 0.17 & 0.17 & $0.32^{* *}$ \\
$T C h o l$ & 0.10 & 0.03 & -0.11 & 0.05 & -0.01 \\
$T G$ & 0.04 & $0.17 *$ & -0.16 & -0.27 & 0.18 \\
$H D L C$ & 0.02 & $-0.17 *$ & -0.02 & 0.03 & -0.23 \\
NHDLC & 0.09 & 0.09 & -0.02 & 0.03 & 0.07 \\
$R L P-C$ & 0.07 & 0.04 & -0.08 & $0.47 *$ & -0.03 \\
$R L P-T G$ & 0.06 & $0.18^{*}$ & -0.07 & $0.42^{*}$ & 0.12 \\
$F B G$ & $0.20 *$ & $0.29 * * *$ & 0.10 & 0.32 & $0.37 * *$ \\
\hline
\end{tabular}

$\neq$ Age is expressed as correlation coefficient, all other values are age-adjusted partial correlations coefficients $* p<0.05, * * p<0.01$, $* * * \mathrm{p}<0.001$. BMI - body mass index, WHR - waist to hip ratio, W - waist, SBP-systolic blood pressure, DBP - diastolic blood pressure, TChol - total cholesterol, TG - triglycerides, HDLC - HDL cholesterol, NHDLC - non-HDL cholesterol, RLP-C - remnant like particles cholesterol, RLP-TG - remnant like particles triglycerides, FBG - fasting blood glycemia.

Table 4. Association of risk factors with IMT CCA, linear regression multivariate analysis.

\begin{tabular}{|c|c|c|c|c|c|}
\hline & $\begin{array}{c}\text { Men } \\
\mathbf{n}=\mathbf{1 2 3}\end{array}$ & $\begin{array}{c}\text { Women all } \\
n=130\end{array}$ & $\begin{array}{c}\text { PremW } \\
n=45\end{array}$ & $\begin{array}{c}\text { NewPostW } \\
n=28\end{array}$ & $\begin{array}{c}\text { PostmW } \\
n=57\end{array}$ \\
\hline Age & $\begin{array}{c}0.006 * * * \\
(0.0012)\end{array}$ & $\begin{array}{c}0.006^{* * *} \\
(0.0008)\end{array}$ & $\begin{array}{c}0.005 * * \\
(0.002)\end{array}$ & $\begin{array}{c}0.014 * * * \\
(0.0023)\end{array}$ & ns \\
\hline$W H R$ & $\mathrm{~ns}$ & ns & $\begin{array}{l}0.318 * * \\
(0.154)\end{array}$ & ns & ns \\
\hline$R L P-C$ & ns & ns & ns & $\begin{array}{l}0.007 * * \\
(0.0023)\end{array}$ & ns \\
\hline$F B G$ & ns & $\begin{array}{c}0.020 * * * \\
(0.0053)\end{array}$ & ns & ns & $\begin{array}{c}0.025 * * * \\
(0.0072)\end{array}$ \\
\hline
\end{tabular}

Data are expressed as regression coefficients (SE). ${ }^{* *} \mathrm{p}<0.01, * * * \mathrm{p}<0.001$. WHR - waist to hip ratio, RLP-C - remnant like particles cholesterol, FBG - fasting blood glycemia.

In PremW and NewPostmW IMT CCA significantly correlated with age. In PostmW, the correlation of IMT CCA with age was not significant. In PremW, IMT CCA was significantly correlated only with WHR. In NewPostmW IMT CCA was significantly correlated with WHR, BMI, RLP-C and RLP-TG. In PostmW, IMT CCA significantly correlated with BMI and FBG.

Linear multiple step regression analysis of association of IMT CCA with risk factors

To evaluate an independent association of IMT CCA with RF, linear multiple step regression analysis was applied. Following RF were put in this model: age,
BMI, WHR, W, SBP, DBP, TChol, TG, HDLC, NHDLC, RLP-C, RLP-TG and FBG. Significant results are shown in Table 4.

In men IMT CCA significantly correlated with age, in women with age and FBG. In men, age accounted for $30 \%$ of IMT CCA values. In women age accounted for $38 \%$ and $\mathrm{FBG}$ for $6 \%$ of IMT CCA values.

In PremW, IMT CCA significantly correlated with age and WHR. Age accounted for $26 \%$ and WHR for $7 \%$ of IMT CCA values. In NewPostmW, IMT CCA significantly correlated with age and RLP-C. Age accounted for $42 \%$ and RLP-C for $17 \%$ of IMT CCA values. In PostmW, IMT CCA significantly correlated with FBG, which accounted for $18 \%$ of IMT CCA values. 
The same analysis was done with exclusion of hysterectomized women. In NewPostmW, the significance of correlation of RLP-C with IMT CCA was unchanged, age accounted for $24 \%$ and RLP-C for $25 \%$ of IMT CCA values. In PostmW, the correlation of IMT CCA with FBG was no more significant (data not shown).

\section{Discussion}

Strong and independent correlation of IMT CCA with RLP-C in newly postmenopausal women was found. This correlation was found neither in men, nor in other groups of women. Significant correlation of IMT CCA with RLP-C was present both in univariate and linear multiple step regression analyses. In addition, when women after hysterectomy were excluded, the RLP-C was the strongest independent predictor of IMT CCA, followed by age. These findings suggest that chance observation is highly improbable and that physiological menopause plays the main role in these relations.

Looking at the risk profile and changes in RF between 1988 and 1996, newly postmenopausal women possessed no exceptional characteristics compared to other groups. Therefore, the most probable explanation is that sudden decrease of estrogens in menopause is responsible for vulnerability of the artery wall and that RLP could be the main RF for progression of atherosclerosis in this period. This hypothesis is supported by already mentioned Framingham study, in which significant association between RPL-C and cardiovascular diseases in women was found (McNamara et al. 2001).

Biological plausibility of these findings cannot be fully explained because exact mechanisms through which menopause affects biological properties of the artery wall are still largely unknown. In animal studies, hormone replacement therapy decreased accumulation of lipoproteins in the artery wall in ovarectomized cynomolgus monkeys, while plasma lipid concentrations remained unchanged (Wagner et al. 1991). In human studies, estrogen receptors were less expressed in endothelial and vascular smooth muscle cells in premenopausal women with ischemic heart disease (Losordo 1994).

None of published studies addressed the effect of RLP on cardiovascular system in women with regard to the time after menopause. However, according to our opinion, early intervention focused at specific lipoprotein subclasses could be of great importance in the prevention of CVD in postmenopausal women. According to our results, women in menopausal transition are most sensitive to particular cardiovascular risk factors, especially smoking (Pitha et al. 2013).

In the univariate analysis, with the exception of age, fasting blood glucose was the only RF correlated with IMT CCA in men and women. In multivariate analysis only in all women and in women postmenopausal for more than 8 years, remained fasting blood glucose significantly correlated with IMT CCA. In women postmenopausal for more than 8 years, after exclusion of hysterectomized women, this association was no more significant.

Because of very high prevalence of diabetes mellitus in postmenopausal women, fasting blood glucose could be one of the strongest predictors for CVD in this population. As already described in experimental settings, increased glycemia could directly influence the metabolism of lipoproteins in the artery wall and promote atherosclerosis (Litwak et al. 1998). Alarming values of BMI, WHR and $\mathrm{W}$ found in postmenopausal women support the hypothesis about the important role of impaired glucose tolerance and insulin resistance in atherogenesis in this group. The prevalence of insulin resistance according to one smaller study (Rosolová et al. 1998) was very high in the Czech population and could be an important cause of increased concentrations of triglycerides and RLP. The fact, that the WHR was after age the strongest independent RF for increased IMT CCA in premenopausal women, stressed the atherogenic role of central obesity and insulin resistance already in premenopause.

In women, using univariate analysis we found significant positive correlation of IMT CCA with TG, RLP-TG and inverse correlation of IMT CCA with HDLC. Looking at the data from women population, concentration of TG between 1988 and 1996 significantly increased and NHDLC did not change. This finding supports in agreement with previously published data (Castelli 1992, Bengtsson et al. 1993) important role of triglycerides as a risk factor for CVD in women.

In contrast to most published studies, plasma lipids, including RLP were not significantly correlated with IMT CCA in men. Absence of the correlation was probably caused by profound decrease in all lipid parameters including triglycerides and non-NHDL cholesterol. Such dynamic changes in lipids, like these seen in our cohort, were not described in other 
observational studies. In study of young Israeli men (soldiers), changes of triglycerides 5 years apart were significant predictor for coronary heart disease (Tirosh et al. 2007). However, also sex seems to exert some influence on lipid profile and in women, but not men increase in circulating triglycerides has been described during middle age (between 40 and 69 years-old) (Kolovou et al. 2009). Selection bias is to our opinion less probable, because no differences in plasma lipids between respondents and non-respondents were found at baseline.

In men, association of IMT CCA with fasting blood glucose was weaker than in women and lost its significance in multivariate analysis. Limitation of this study is the absence of data about fasting blood glucose at the beginning. Therefore, we cannot exclude selection, causing underrepresenation of these men with impaired glucose tolerance or diabetes mellitus at the second examination.

As expected, IMT CCA was strongly associated with age in almost all groups under study. The only exception was the oldest group of postmenopausal women. In this group, the impact of age as a RF for atherosclerosis was probably attenuated.

With the exception of waist circumference, waist to hip ratio and diastolic blood pressure, there were no significant differences between men and women in cardiovascular risk factors. This finding underlines the fact that studied women (63\% postmenopausal) were at extremely high risk for cardiovascular diseases. Indeed, no difference was found in IMT CCA between men and women.

In summary, the main advantage of this study was randomly selected population sample with high respondence rate. One limitation of the study was relatively low number of participants. Another limitation was the absence of data about changes in concentrations of remnant-like particles and fasting blood glucose.

However, despite relatively low number of participants, strong and independent association between RLP-C and IMT CCA was found in postmenopausal women. This association was limited to the period of several years after menopause. We can conclude that remnant-like particle cholesterol is in this population more important measurable and modifiable RF than total, LDL or non-HDL cholesterol. This study could stimulate intervention trials focused on reduction of RLP in highrisk postmenopausal women already early after beginning of menopause.

\section{Conflict of Interest}

There is no conflict of interest.

\section{Acknowledgements}

This project was supported through the Internal Grant Agency of the Ministry of Health, Czech Republic (NT 14008-3/2013 and NT 13151-3/2013) and by the research project $\mathrm{MH} \mathrm{CZ} \mathrm{-} \mathrm{DRO} \mathrm{(Institute} \mathrm{for} \mathrm{Clinical} \mathrm{and}$ Experimental Medicine - IKEM, IN 00023001) of the Ministry of Health of the Czech Republic.

\section{References}

AUSTIN MA: Epidemiology of hypertriglyceridemia and cardiovascular disease. Am J Cardiol 83: 13F-16F, 1999.

BENGTSSON C, BJORKELUND C, LAPIDUS L, LISSNER L: Associations of serum lipid concentrations and obesity with mortality in women: 20 year follow up of participants in prospective population study in Gothenburg, Sweden. BMJ 307: 1385-1388, 1993.

BOQUiST S, RUOTOLO G, TANG R, BJÖRKEGREN J, BOND MG, DE FAIRE U, KARPE F, HAMSTEN A: Alimentary lipemia, postprandial triglyceride-rich lipoproteins, and common carotid intima-media thickness in healthy, middle-aged men. Circulation 100: 723-728, 1999.

CASTELLI WP: Epidemiology of triglycerides: a view from Framingham. Am J Cardiol 70: 3H-9H, 1992.

CÍFKOVÁ R, SKODOVÁ Z, BRUTHANS J, ADÁMKOVÁ V, JOZÍFOVÁ M, GALOVCOVÁ M, WOHLFAHRT P, KRAJCOVIECHOVÁ A, POLEDNE R, STÁVEK P, LÁNSKÁ V: Longitudinal trends in major cardiovascular risk factors in the Czech population between 1985 and 2007/8. Czech MONICA and Czech post-MONICA. Atherosclerosis 211: 676-668, 2010.

HAVEL RJ: Postprandial hyperlipidemia and remnant lipoproteins. Curr Opin Lipidol 5: 102-109, 1994.

HOKANSON JE, AUSTIN MA: Plasma triglyceride level is a risk factor for cardiovascular disease independent of highdensity lipoprotein cholesterol level: a metaanalysis of population-based prospective studies. J Cardiovasc Risk $\mathbf{3}$ : 213-219, 1996. 
INOUE T, SANIABADI AR, MATSUNAGA R, HOSHI K, YAGUCHI I, MOROOKA S: Impaired endotheliumdependent acetylcholine-induced coronary artery relaxation in patients with high serum remnant lipoprotein particles. Atherosclerosis 139: 363-367, 1998.

JEPPESEN J, HEIN HO, SUADICANI P, GYNTELBERG F: Triglyceride concentration and ischemic heart disease. An eight-year follow-up in the Copenhagen Male Study. Circulation 97: 1029-1036, 1998.

KARPE F, BOQUIST S, TANG R, BOND GM, DE FAIRE U, HAMSTEN A: Remnant lipoproteins are related to intimamedia thickness of the carotid artery independently of LDL cholesterol and plasma triglycerides. J Lipid Res $\mathbf{4 2}$ : $17-21,2001$.

KOLOVOU GD, ANAGNOSTOPOULOU KK, DAMASKOS DS, BILIANOU HI, MIHAS C, MILIONIS HJ, KOSTAKOU PM, COKKINOS DV: Gender differences in the lipid profile of dyslipidemic subjects. Eur J Intern Med 20: 145-151, 2009.

KUGIYAMA K, DOI H, TAKAZOE K, KAWANO H, SOEJIMA H, MIZUNO Y, TSUNODA R, SAKAMOTO T, NAKANO T, NAKAJIMA K, OGAWA H, SUGIYAMA S, YOSHIMURA M, YASUE H: Remnant lipoprotein levels in fasting serum predict coronary events in patients with coronary artery disease. Circulation 99: 28582860, 1999.

LIBBY P: Triglycerides on the rise: should we swap seats on the seesaw? Eur Heart J 36: 774-776, 2015.

LITWAK KN, CEFALU WT, WAGNER JD: Chronic hyperglycemia increases arterial low-density lipoprotein metabolism and atherosclerosis in cynomolgus monkeys. Metabolism 47: 947-954, 1998.

LOSORDO DW, KEARNEY M, KIM EA, JEKANOWSKI J, ISNER JM: Variable expression of the estrogen receptor in normal and atherosclerotic coronary arteries of premenopausal women. Circulation 89: 1501-1510, 1994.

MCNAMARA JR, SHAH PK, NAKAJIMA K, CUPPLES LA, WILSON PW, ORDOVAS JM, SCHAEFER EJ: Remnant-like (RLP) cholesterol is an independent cardiovascular risk factor in women: results from the Framingham Heart Study. Atherosclerosis 154: 229-236, 2001.

MILLER M, SEIDLER A, MOALEMI A, PEARSON TA: Normal triglyceride levels and coronary artery disease events: The Baltimore Coronary Observational Long-Term Study. J Am Coll Cardiol 31: 1252-1257, 1998.

NAKAJIMA K, OKAZAKI M, TANAKA A, PULLINGER CR, WANG T, NAKANO T, ADACHI M, HAVEL RJ: Separation and determination of remnant-like particles in human serum using monoclonal antibodies to Apo B-100 and Apo A-1. J Clin Ligand Assay 19: 177-183, 1996.

NORDESTGAARD BG, VARBO A: Triglycerides and cardiovascular disease. Lancet 384: 626-635, 2014.

PIT'HA J, KRAJÍCKOVÁ D, CÍFKOVÁ R, HUBÁCEK J, PETRZÍLKOVÁ Z, HEJL Z, STÁVEK P, SKIBOVÁ J, POLEDNE R: Intima-media thickness of carotid arteries in borderline hypertensives. J Neuroimaging 9: 19-22, 1999.

PITHA J, LESNÁ K, SEKERKOVA A, POLEDNE R, KOVÁŘ J, LEJSKOVÁ M, DVOŘÁKOVÁ H, ADÁMKOVÁ S, LÁNSKÁ V, BOBAK M: Menopausal transition enhances the atherogenic risk of smoking in middle aged women. Int J Cardiol 168: 190-196, 2013.

ROSOLOVÁ H, MAYER O JR, ŠIMON J, SEFRNA F: Detection of risk of insulin resistance in the population (in Czech). Čas Lék Česk 137: 80-83, 1998.

TAKEICHI S, NAKAJIMA Y, OSAWA M, YUKAWA N, SAITO T, SETO Y, NAKANO T, ADACHI M, JITSUKATA K, HORIUCHI K, WANG T, NAKAJIMA K: The possible role of remnant-like particles as a risk factor for sudden cardiac death. Int J Legal Med 110: 213-219, 1997.

TIROSH A, RUDICH A, SHOCHAT T, TEKES-MANOVA D, ISRAELI E, HENKIN Y, KOCHBA I, SHAI I: Changes in triglyceride levels and risk for coronary heart disease in young men. Ann Intern Med 18: 377-385, 2007.

VARBO A, NORDESTGAARD BG: Remnant cholesterol and ischemic heart disease. Curr Opin Lipidol 25: 266-273, 2014.

WAGNER JD, CLARKSON TB, ST CLAIR RW, SCHWENKE DC, SHIVELY CA, ADAMS MR: Estrogen and progesterone replacement therapy reduces low density lipoprotein accumulation in the coronary arteries of surgically postmenopausal cynomolgus monkeys. J Clin Invest 88: 1995-2002, 1991. 